\title{
Spin-flip inelastic electron tunneling spectroscopy in atomic chains
}

\author{
Aaron Hurley, Nadjib Baadji, and Stefano Sanvito \\ School of Physics and Centre for Research on Adaptive Nanostructures and Nanodevices (CRANN), Trinity College, Dublin 2, Ireland
}

(Received 15 March 2011; revised manuscript received 16 June 2011; published 25 July 2011)

\begin{abstract}
We present a theoretical study of the spin transport properties of monoatomic magnetic chains with a focus on the spectroscopical features of the $I-V$ curve associated with spin-flip processes. Our calculations are based on the $s$ - $d$ model for magnetism with the electron transport treated at the level of the nonequilibrium Green's function formalism. Inelastic spin-flip scattering processes are introduced perturbatively via the first Born approximation, and an expression for the associated self-energy is derived. The computational method is then applied to describe the $I-V$ characteristics and its derivatives of one-dimensional chains of $\mathrm{Mn}$ atoms, and the results are then compared to available experimental data. We find a qualitative and quantitative agreement between the calculated and the experimental conductance spectra. Significantly we are able to describe the relative intensities of the spin excitation features in the $I-V$ curve, by means of a careful analysis of the spin transition selection rules associated with the atomic chains.
\end{abstract}

DOI: 10.1103/PhysRevB.84.035427

PACS number(s): 73.40.Gk, 68.37.Ef, 85.75.Mm, 71.70.Gm

\section{INTRODUCTION}

Inelastic electron tunneling spectroscopy (IETS) has become an important tool for investigating the elementary excitations of nanoscale systems. ${ }^{1}$ An excitation manifests itself as an abrupt change in the differential conductance of a two-probe device as the voltage sweeps across the excitation energy. This is the result of the opening of a new inelastic transport channel for the electron tunneling. As the energy of the probed excitation sets the critical voltage and the temperature range where to observe IETS, it is not surprising that the first experiments were all related to molecular vibrations of relatively high energy. ${ }^{2}$

More recently the continuous advances in low-temperature scanning tunneling microscopy (STM) have enabled the detection of excitations of different origin, namely, those related to the spin degree of freedom. This different type of spectroscopy is usually named spin-flip IETS (SF-IETS). The first measurements of SF-IETS were for single atoms randomly deposited on surfaces. ${ }^{3}$ However, STM techniques also open the possibility of assembling and manipulating entire nanostructures ${ }^{4}$ and of positioning magnetic ions on a surface at a desired position. This enables the construction of atomic magnetic nanostructures and the study of the complex magnetic excitations, resulting from the exchange interaction between the magnetic ions and the substrate, and also between the magnetic atoms themselves. Such a unique fabrication capability has produced a surge of experimental studies on the spin excitations of magnetic nanostructures. These comprise the investigation of the conductance spectra of individual atomic spins, ${ }^{5}$ of Kondo-screened magnetic dimers, ${ }^{6}$ and of multiple chains of $\mathrm{Mn}$ atoms. ${ }^{7}$ Interestingly similar investigations have now been extended to molecular chains composed of Co-phthalocyanines. ${ }^{8}$

The recent rapid growth in the experimental activity has been matched by an equally fast explosion of theoretical works. A general and now standard approach to calculating the conductance spectra of the various possible magnetic nanostructures is that of combining a master equation solver for the quantum transport problem with a model Hamiltonian describing the magnetic interaction. ${ }^{9}$ This is an intrinsic many-body approach, which in principle contains all the ingredients needed for solving the problem, once the various transfer rates are known. As such it usually requires a large number of parameters to be predictive. An alternative strategy consists of treating the problem at the single-particle level, by using a tunnel Hamiltonian and/or the standard TersoffHamann description ${ }^{10}$ for the STM current. ${ }^{11-14}$ This second class of computational schemes appears more amenable to be implemented together with first-principles electron transport methods. However, it still remains a hybrid theory, where the dynamic part of the problem needs to be approached at the model Hamiltonian level, although the level of sophistication may include effects related to current generated nonequilibrium spin populations. ${ }^{15,16}$

A standard theoretical approach to quantum transport is represented by the nonequilibrium Green's function (NEGF) formalism, ${ }^{17,18}$ whose mean-field version can be combined with state-of-the-art electronic structure theories to produce efficient and predictive algorithms. ${ }^{19,20}$ To the best of our knowledge, this is, to date, the only fully quantitative quantum transport approach capable of scaling to large systems, ${ }^{21}$ i.e., it is the only one capable of performing simulations for materials sets of current technological relevance. Importantly for this discussion, inelastic contributions to the elastic current can also be included within the NEGF formalism. In the case of scattering to phonons the problem is usually treated perturbatively by constructing an appropriate self-energy at the level of either the first (1BA) or the self-consistent Born approximation (SCBA). ${ }^{22}$ To the best of our knowledge, a similar approach to the case of spin excitations is currently not available.

There are two main reasons for this gap. On the one hand, the adiabatic separation between the electronic degrees of freedom and those responsible for the inelastic excitations are well defined in the case of nuclear motion (phonons) but less clear in the case of spins, since even extremely localized spins have full electronic origin. ${ }^{23}$ On the other hand, it is also unclear whether the perturbative approach is valid for spins, 


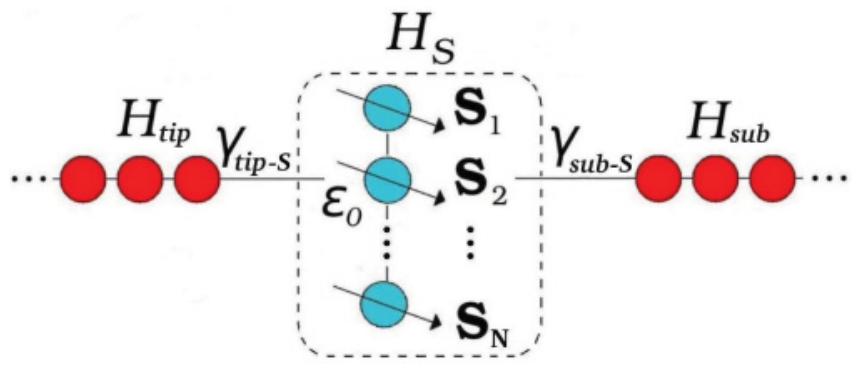

FIG. 1. (Color online) Schematic representation of the device investigated in this work. A scattering region, comprising $N$ spincarrying atoms (light-blue circles) and described by the Hamiltonian $H_{\mathrm{S}}$, is sandwiched in between two semi-infinite electrodes (red circles). These mimic the substrate and the tip in a typical STM experiment. The electrodes are nonspin polarized, and they are described by the Hamiltonian $H_{\text {sub }}$ and $H_{\text {tip }}$. In the scattering region the transport electrons are exchange coupled to local quantum spins $\mathbf{S}_{i}$.

i.e., whether a suitable expansion parameter can be found. As such, as far as we know, an expression for a self-energy describing inelastic spin-flip events has not been derived so far.

In the present paper we undertake this challenge and formulate a perturbative theory of spin-flip spectroscopy based on the NEGF formalism for electron transport. Our theoretical analysis is based on a tight-binding Hamiltonian for the transport electrons, which are locally exchange coupled to quantum spins. As such our formulation works by still assuming an adiabatic separation between the transport electrons and the local spins, and it is constructed over the $s-d$ model for magnetism. ${ }^{24}$ We then proceed in constructing an appropriate self-energy for the spin degrees of freedom at the 1BA level (note that for this particular problem little differences are introduced by extending the treatment to the SCBA) and use this in the standard NEGF scheme for transport. Our methodology is then applied to describing SF-IETS in atoms and atomic chains and results are favorably compared with experiments.

\section{INTERACTION HAMILTONIAN AND THE SCATTERING SELF-ENERGY}

The typical experimental setup considered in this work is that of a STM measurement, i.e., it comprises a STM tip positioned above one of the atoms of a magnetic nanostructure, which in turn is weakly coupled to a metallic substrate across an insulating barrier.

We model this system by using a pair of noninteracting semi-infinite leads that are separated by a scattering region as outlined in Fig. 1. The left-hand side lead, the scattering region, and the right-hand side lead represent respectively the STM tip, the spin-coupled nanostructure, and the substrate and they are described by the Hamiltonian $H_{\text {tip }}, H_{\mathrm{S}}$, and $H_{\text {sub }}$. For simplicity we assume an identical electronic structure for both the leads (i.e., they are made of the same material), which we describe by a one orbital per site tight-binding Hamiltonian with nearest-neighbor interaction.
The scattering region consists of a one-dimensional chain of $N$ atoms. Each $i$ th atom carries a quantum mechanical spin $\mathbf{S}_{i}$ and is characterized by an on-site energy $\varepsilon_{0}$. We assume that the tip and substrate can only couple to one atom at the time in the scattering region, and we describe such a coupling by the hopping integrals $\gamma_{\text {tip-S }}$ and $\gamma_{\text {sub-S }}$, respectively. This means that the electronic states of the scattering region are broadened by the interaction with the electrodes by $\Gamma_{\text {tip-S }}=\gamma_{\text {tip-S }}^{2} / \gamma_{0}$ and $\Gamma_{\text {sub-S }}=\gamma_{\text {sub-S }}^{2} / \gamma_{0}$, where $\gamma_{0}$ is the hopping parameter in the leads. We assume that $\varepsilon_{0} \gg \Gamma_{\text {tip-S }}$ (and also $\varepsilon_{0} \gg \Gamma_{\text {sub-S }}$ ), leading to a constant density of states at the Fermi energy $\left(E_{\mathrm{F}}\right)$. This assumption has two main consequences. On the one hand, we can neglect the electronic interaction among the atoms in the scattering region (i.e., the creation of bonding and antibonding levels), as this will generate states far enough from the Fermi energy to ensure a constant density of states. On the other hand, we can simplify the interaction between the atoms in the chain and the substrate to an electronic coupling to a single atom, as additional coupling (as far as it remains weak) will not introduce additional density of states around $E_{\mathrm{F}}$.

The Hamiltonian of the scattering region contains three parts $H_{\mathrm{S}}=H_{\mathrm{e}}+H_{\mathrm{sp}}+H_{\mathrm{e}-\mathrm{sp}}$, where $H_{\mathrm{e}}$ is the tight-binding electronic part, $H_{\mathrm{sp}}$ is the spin part, and $H_{\mathrm{e}-\mathrm{sp}}$ describes the electron-spin interaction. More explicitly these three components write respectively as

$$
\begin{gathered}
H_{\mathrm{e}}=\varepsilon_{0} \sum_{\lambda \alpha} c_{\lambda \alpha}^{\dagger} c_{\lambda \alpha}, \\
H_{\mathrm{sp}}=2 J_{d d} \sum_{\lambda}^{N-1} \mathbf{S}_{\lambda} \cdot \mathbf{S}_{\lambda+1}+\sum_{\lambda}^{N}\left\{g \mu_{\mathrm{B}} \mathbf{B} \cdot \mathbf{S}_{\lambda}+D\left(S_{\lambda}^{z}\right)^{2}\right. \\
\left.+E\left[\left(S_{\lambda}^{x}\right)^{2}-\left(S_{\lambda}^{y}\right)^{2}\right]\right\}, \\
H_{\mathrm{e}-\mathrm{sp}}=J_{s d} \sum_{\lambda \alpha, \alpha^{\prime}}\left(c_{\lambda \alpha}^{\dagger}\left[\sigma_{\lambda}\right]_{\alpha \alpha^{\prime}} c_{\lambda \alpha^{\prime}}\right) \cdot \mathbf{S}_{\lambda} .
\end{gathered}
$$

The electronic part consists only of an on-site potential, i.e., we neglect electron hopping between the sites [the electron ladder operators $c_{\lambda \alpha}^{\dagger} / c_{\lambda \alpha}$ create/annihilate an electron at site $\lambda$ with spin $\alpha(=\uparrow, \downarrow)]$.

We model the spin-spin interaction between the localized spins $\left\{\mathbf{S}_{\lambda}\right\}$ by a nearest-neighbor Heisenberg Hamiltonian with coupling strength $J_{d d}$. Furthermore, we include interaction with an external magnetic field $\mathbf{B}$ ( $\mu_{\mathrm{B}}$ is the Bohr magneton and $g$ the gyromagnetic ratio) and both uniaxial and transverse anisotropy of magnitude $D$ and $E$, respectively. ${ }^{6,25}$

The electron-spin interaction Hamiltonian is constructed within the $s$ - $d$ model, ${ }^{24}$ where the transport electron $s$ are locally exchanged coupled to quantum spins $\left\{\mathbf{S}_{\lambda}\right\}$ (" $d$ " indicates that the local moments originate from the atomic $d$ shell). The electronic spins are described by the operator $c_{\lambda \alpha}^{\dagger}\left[\sigma_{\lambda}\right]_{\alpha \alpha^{\prime}} c_{\lambda \alpha^{\prime}}$, with $\sigma$ being the vector of Pauli matrices. The use of the $s$ - $d$ model means that we implicitly assume an adiabatic separation between the transport electrons and those forming the local spins. As a consequence we can define a single interaction parameter $J_{s d}$, which can be used to develop a perturbation theory (note, however, that $J_{s d}$ is not 
the perturbation expansion parameter, as it will be explained later). Such an approximation is valid in the limit of weak electronic coupling between the electron reservoirs and the scattering region, as in the case of the STM measurements that we aim to describe here. Note that going beyond such an approximation will require formulating an entirely electronic theory for inelastic spin transport. An Anderson-like impurity model can be a starting Hamiltonian for such a task, ${ }^{26}$ but this will require abandoning the perturbative approach and it is outside the scope of the present work.

We now proceed to deriving the perturbation theory in the electron-spin interaction (note that $H_{\mathrm{e}}$ and $H_{\text {sp }}$ independently can be easily diagonalized). Our strategy is that of first constructing the many-body Green's function at the level of $1 \mathrm{BA},{ }^{27-30}$ and then, by Dyson's equation, to evaluate the interacting self-energy. In particular, we follow closely the procedure laid out in Ref. 27. Since we consider a nonequilibrium situation at zero temperature our starting point is the Keldysh ${ }^{17}$ contour-ordered single-body Green's function in the many-body ground state,

$$
\left[G\left(\tau, \tau^{\prime}\right)\right]_{\sigma \sigma^{\prime}}=-i\left\langle\left|T_{C}\left\{c_{\sigma}(\tau) c_{\sigma^{\prime}}^{\dagger}\left(\tau^{\prime}\right)\right\}\right|\right\rangle,
$$

where the time average is performed over the full interacting ground state |\rangle (note that for clarity we have dropped the site index $\lambda$, which will be explicitly included only where necessary). Equation (4) can be expanded up to the $n$th order in the interaction Hamiltonian $H_{\mathrm{e}-\text { sp }}$ as

$$
\begin{aligned}
{\left[G\left(\tau, \tau^{\prime}\right)\right]_{\sigma \sigma^{\prime}}=} & \sum_{n} \frac{(-i)^{n+1}}{n !} \int_{C} d \tau_{1} \cdots \int_{C} d \tau_{n} \\
& \times \frac{\left\langle 0\left|T_{C}\left\{H_{\mathrm{e}-\mathrm{sp}}\left(\tau_{1}\right) \cdots H_{\mathrm{e}-\mathrm{sp}}\left(\tau_{n}\right) c_{\sigma}(\tau) c_{\sigma^{\prime}}^{\dagger}\left(\tau^{\prime}\right)\right\}\right| 0\right\rangle}{U(-\infty,-\infty)},
\end{aligned}
$$

where $U$ is the time-evolution unitary operator and the time averages are now over the known noninteracting $\left(J_{s d}=0\right)$ ground state $|0\rangle$. The time integration over $\tau$ is ordered on the contour $C$ going from $-\infty$ to $+\infty$ and then returning from $+\infty$ to $-\infty$, since the ground state of the nonequilibrium system can only be defined at $-\infty .{ }^{31}$ If the expansion is truncated to the first order, one obtains a Zeeman-like term which can be neglected as long as $\varepsilon_{0} \gg \gamma_{\text {tip-S(sub-S). The }}$ first contribution of interest then appears at the second order. This can be obtained by inserting the explicit expression for $H_{\mathrm{e}-\mathrm{sp}}(t)[$ Eq. (3)] into Eq. (4),

$$
\begin{aligned}
{\left[G\left(\tau, \tau^{\prime}\right)\right]_{\sigma \sigma^{\prime}}^{(2)}=} & \frac{(-i)^{3}}{2 !} J_{s d}^{2} \sum_{i, \alpha, \alpha^{\prime}, j, \beta, \beta^{\prime}} \int_{C} d \tau_{1} \int_{C} d \tau_{2}\langle 0| T_{C}\left\{c_{\sigma}(\tau)\right. \\
& \left.\times c_{\alpha}^{\dagger}\left(\tau_{1}\right) c_{\alpha^{\prime}}\left(\tau_{1}\right) c_{\beta}^{\dagger}\left(\tau_{2}\right) c_{\beta^{\prime}}\left(\tau_{2}\right) c_{\sigma^{\prime}}^{\dagger}\left(\tau^{\prime}\right)\right\}|0\rangle \\
& \times\left\langle 0\left|T_{C}\left\{S^{i}\left(\tau_{1}\right) S^{j}\left(\tau_{2}\right)\right\}\right| 0\right\rangle\left[\sigma^{i}\right]_{\alpha \alpha^{\prime}}\left[\sigma^{j}\right]_{\beta \beta^{\prime}}, \quad(6)
\end{aligned}
$$

where the indices $i$ and $j$ run over the Cartesian coordinates $x, y$, and $z$ (not to be confused with the site index).

A full contour-ordered expansion must now be performed on both the electron bracket and the spin bracket. The electron bracket has six different time-ordering combinations, which are explicitly listed in Eq. (7) below:

$$
\begin{aligned}
\langle 0| T_{C} & \left\{c_{\sigma}(\tau) c_{\alpha}^{\dagger}\left(\tau_{1}\right) c_{\alpha^{\prime}}\left(\tau_{1}\right) c_{\beta}^{\dagger}\left(\tau_{2}\right) c_{\beta^{\prime}}\left(\tau_{2}\right) c_{\sigma^{\prime}}^{\dagger}\left(\tau^{\prime}\right)\right\}|0\rangle \\
= & \left\langle 0\left|T_{C}\left\{c_{\sigma}(\tau) c_{\alpha}^{\dagger}\left(\tau_{1}\right)\right\}\right| 0\right\rangle\left\langle 0\left|T_{C}\left\{c_{\alpha^{\prime}}\left(\tau_{1}\right) c_{\beta}^{\dagger}\left(\tau_{2}\right)\right\}\right| 0\right\rangle \\
& \times\left\langle 0\left|T_{C}\left\{c_{\beta^{\prime}}\left(\tau_{2}\right) c_{\sigma^{\prime}}^{\dagger}\left(\tau^{\prime}\right)\right\}\right| 0\right\rangle+\left\langle 0\left|T_{C}\left\{c_{\sigma}(\tau) c_{\beta}^{\dagger}\left(\tau_{2}\right)\right\}\right| 0\right\rangle \\
& \times\left\langle 0\left|T_{C}\left\{c_{\alpha^{\prime}}\left(\tau_{1}\right) c_{\sigma^{\prime}}^{\dagger}\left(\tau^{\prime}\right)\right\}\right| 0\right\rangle\left\langle 0\left|T_{C}\left\{c_{\alpha}\left(\tau_{2}\right) c_{\beta^{\prime}}^{\dagger}\left(\tau_{1}\right)\right\}\right| 0\right\rangle \\
& +\left\langle 0\left|T_{C}\left\{c_{\sigma}(\tau) c_{\alpha}^{\dagger}\left(\tau_{1}\right)\right\}\right| 0\right\rangle\left\langle 0\left|T_{C}\left\{c_{\alpha^{\prime}}\left(\tau_{1}\right) c_{\sigma^{\prime}}^{\dagger}\left(\tau^{\prime}\right)\right\}\right| 0\right\rangle \\
& \times\left\langle 0\left|T_{C}\left\{c_{\beta}^{\dagger}\left(\tau_{2}\right) c_{\beta^{\prime}}\left(\tau_{2}\right)\right\}\right| 0\right\rangle+\left\langle 0\left|T_{C}\left\{c_{\sigma}(\tau) c_{\beta}^{\dagger}\left(\tau_{2}\right)\right\}\right| 0\right\rangle \\
& \times\left\langle 0\left|T_{C}\left\{c_{\beta^{\prime}}\left(\tau_{2}\right) c_{\sigma^{\prime}}^{\dagger}\left(\tau^{\prime}\right)\right\}\right| 0\right\rangle\left\langle 0\left|T_{C}\left\{c_{\alpha}^{\dagger}\left(\tau_{1}\right) c_{\alpha^{\prime}}\left(\tau_{1}\right)\right\}\right| 0\right\rangle \\
& +\left\langle 0\left|T_{C}\left\{c_{\sigma}(\tau) c_{\sigma^{\prime}}^{\dagger}\left(\tau^{\prime}\right)\right\}\right| 0\right\rangle\left\langle 0\left|T_{C}\left\{c_{\alpha}^{\dagger}\left(\tau_{1}\right) c_{\alpha^{\prime}}\left(\tau_{1}\right)\right\}\right| 0\right\rangle \\
& \times\left\langle 0\left|T_{C}\left\{c_{\beta}^{\dagger}\left(\tau_{2}\right) c_{\beta^{\prime}}\left(\tau_{2}\right)\right\}\right| 0\right\rangle-\left\langle 0\left|T_{C}\left\{c_{\sigma}(\tau) c_{\sigma^{\prime}}^{\dagger}\left(\tau^{\prime}\right)\right\}\right| 0\right\rangle \\
& \times\left\langle 0\left|T_{C}\left\{c_{\alpha^{\prime}}\left(\tau_{1}\right) c_{\beta}^{\dagger}\left(\tau_{2}\right)\right\}\right| 0\right\rangle\left\langle 0\left|T_{C}\left\{c_{\beta^{\prime}}\left(\tau_{2}\right) c_{\alpha}^{\dagger}\left(\tau_{1}\right)\right\}\right| 0\right\rangle .
\end{aligned}
$$

The first and the second terms represent Fock-like Feynman diagrams [see Fig. 2(b)], while the third and the fourth ones correspond to Hartree-like ones (note that Hartree-like diagrams vanish because of the spin selection rules, as discussed later in this section). Both these pairs are equal under the exchange of the indexes. Finally, the last two combinations can be eliminated since they represent unconnected Feynman diagrams which vanish in the averaging process. ${ }^{27}$ This leaves us with a simplified expression which, when compared to Eq. (5), gives us

$$
\begin{aligned}
\langle 0| T_{C} & \left\{c_{\sigma}(\tau) c_{\alpha}^{\dagger}\left(\tau_{1}\right) c_{\alpha^{\prime}}\left(\tau_{1}\right) c_{\beta}^{\dagger}\left(\tau_{2}\right) c_{\beta^{\prime}}\left(\tau_{2}\right) c_{\sigma^{\prime}}^{\dagger}\left(\tau^{\prime}\right)\right\}|0\rangle \\
= & 2 i^{3} \delta_{\sigma \alpha} \delta_{\alpha^{\prime} \beta} \delta_{\beta^{\prime} \sigma^{\prime}}\left[G_{0}\left(\tau, \tau_{1}\right)\right]_{\sigma \sigma}\left[G_{0}\left(\tau_{1}, \tau_{2}\right)\right]_{\alpha^{\prime} \alpha^{\prime}}\left[G_{0}\left(\tau_{2}, \tau^{\prime}\right)\right]_{\sigma^{\prime} \sigma^{\prime}} \\
& +2 i^{3} \delta_{\sigma \alpha} \delta_{\alpha^{\prime} \sigma^{\prime}} \delta_{\beta \beta^{\prime}}\left[G_{0}\left(\tau, \tau_{1}\right)\right]_{\sigma \sigma}\left[G_{0}\left(\tau_{1}, \tau^{\prime}\right)\right]_{\sigma^{\prime} \sigma^{\prime}}\left[G_{0}\left(\tau_{2}, \tau_{2}\right)\right]_{\beta \beta} .
\end{aligned}
$$

In this case, since the averaging bracket is over the noninteracting ground state, $G_{0}$ represents the unperturbed electronic Green's function and can be calculated exactly.

We then return to Eq. (6) and evaluate the spin bracket. The ground state of the noninteracting spin system alone can be found by diagonalizing exactly $H_{\mathrm{sp}}$. This is achieved by constructing the full basis $\{|n\rangle\}$ where $n=-S,-S+$ $1, \ldots,+S$. Note that in the discussion of the results we will

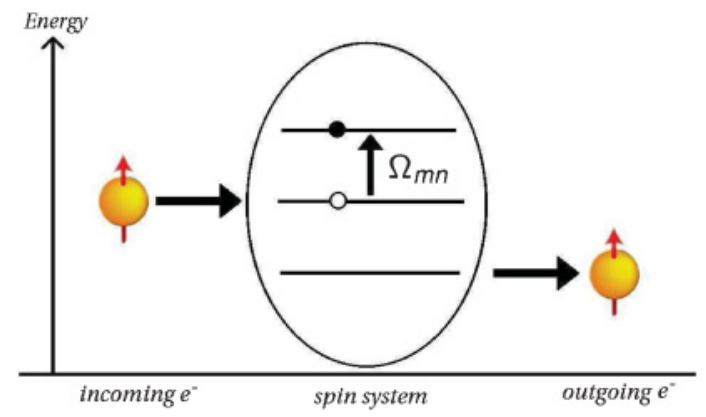

FIG. 2. (Color online) (A) Schematic representation of the inelastic process described by the greater self-energy $\Sigma_{\text {int }}^{>}$. An incoming electron scatters against a localized spin and decreases its energy by $\Omega_{m n}$. This is transfer to the local spin system, which undergoes a spin transition $|n\rangle \rightarrow|m\rangle$. (B) Feynman Fock-like diagram describing the interaction in the time domain. 
keep labeling the eigenvalues of the spin Hamiltonian with the $z$ component of the total spin $S$, which in general is not a good quantum number because of the presence of the transverse anisotropy. However, such an anisotropy is small so that our notation remains approximately valid. The resulting eigenvectors $|m\rangle$ and eigenvalues $E_{m}$ satisfy the Schrödinger equation $H_{\mathrm{sp}}|m\rangle=\varepsilon_{m}|m\rangle$, and they can be used to rewrite the operators $S^{i}(\tau)$, for $i=\{x, y, z\}$, as

$$
S^{i}(\tau)=\sum_{m, n}\left\langle m\left|S^{i}\right| n\right\rangle d_{m}^{\dagger}(\tau) d_{n}(\tau) .
$$

Here $d_{n}$ is a destruction operator for a quasiparticle of the spin system. The quasiparticles are then assumed to be fermionic in nature so that they obey the anticommutation rules $\left[d_{m}^{\dagger}, d_{n}\right]=$ $\delta_{m n}$ and $\left[d_{m}^{\dagger}, d_{n}^{\dagger}\right]=\left[d_{m}, d_{n}\right]=0$. Such an assumption is valid as long as the excitations considered are always around the ground state, i.e., under the condition that the spin system can always efficiently relax back to the ground state between spin-flip events. We can then define a contour-ordered spin Green's function as follows:

$$
\left[D\left(\tau, \tau^{\prime}\right)\right]_{n, m}=-i\left\langle\left|T_{C}\left\{d_{n}(\tau) d_{m}^{\dagger}\left(\tau^{\prime}\right)\right\}\right|\right\rangle .
$$

By inserting the expressions in Eqs. (8) and (9) into the spin bracket and by computing the time-ordered contraction, we finally obtain

$$
\begin{aligned}
\left\langle 0\left|T_{C}\left\{S^{i}\left(\tau_{1}\right) S^{j}\left(\tau_{2}\right)\right\}\right| 0\right\rangle= & -\sum_{m, n}\left\langle m\left|S^{i}\right| n\right\rangle\left\langle n\left|S^{j}\right| m\right\rangle \\
& \times\left[D_{0}\left(\tau_{1}, \tau_{2}\right)\right]_{n, n}\left[D_{0}\left(\tau_{2}, \tau_{1}\right)\right]_{m, m} .
\end{aligned}
$$

The set of equations ((8)) and (10) can now be incorporated into the expression for the second-order contribution to the many-body Green's function [Eq. (6)]. Then, by using Dyson's equation, ${ }^{27}$ one can finally write the second-order contribution to the interacting self-energy, which reads

$$
\begin{aligned}
{\left[\Sigma_{\text {int }}\left(\tau_{1}, \tau_{2}\right)\right]_{\sigma \sigma^{\prime}}^{(2)}=} & -J_{s d}^{2} \sum_{i, j, \beta}\left\{\left[\sigma^{i}\right]_{\sigma \beta}\left[\sigma^{j}\right]_{\beta \sigma^{\prime}}+\left[\sigma^{i}\right]_{\sigma \sigma^{\prime}}\left[\sigma^{j}\right]_{\beta \beta}\right\} \\
& \times\left[G_{0}\left(\tau_{1}, \tau_{2}\right)\right]_{\beta \beta} \sum_{m, n}\left\langle m\left|S^{i}\right| n\right\rangle\left\langle n\left|S^{j}\right| m\right\rangle \\
& \times\left[D_{0}\left(\tau_{1}, \tau_{2}\right)\right]_{n, n}\left[D_{0}\left(\tau_{2}, \tau_{1}\right)\right]_{m, m} .
\end{aligned}
$$

If we now assume that the ground-state electronic spin levels are degenerate, i.e., $\left[G_{0}\right]_{\uparrow \uparrow}=\left[G_{0}\right]_{\downarrow \downarrow}$, then the only quantity of interest is the trace of the self-energy over spin indices. By performing such a trace, the spin-independent self-energy finally reads

$$
\begin{aligned}
\Sigma_{\text {int }}\left(\tau_{1}, \tau_{2}\right)^{(2)}= & -2 J_{s d}^{2} \sum_{i, m, n}\left|\left\langle m\left|S^{i}\right| n\right\rangle\right|^{2} G_{0}\left(\tau_{1}, \tau_{2}\right) \\
& \times\left[D_{0}\left(\tau_{1}, \tau_{2}\right)\right]_{n, n}\left[D_{0}\left(\tau_{2}, \tau_{1}\right)\right]_{m, m},
\end{aligned}
$$

where we have used the results $\operatorname{Tr}\left[\sigma^{i} \sigma^{j}\right]=\delta_{i j}$ and $\operatorname{Tr}\left[\sigma^{i}\right]=$ 0 . Note that, at variance with the phonon case, the relation $\operatorname{Tr}\left[\sigma^{i}\right]=0$ guarantees that the Hartree-like diagrams do not contribute to the self-energy.

At this point we can calculate the real-time quantities, such as the lesser (greater) self-energies, by using the Langreth's theorem for time ordering over the contour $\tau_{1} \in C_{1}\left(C_{2}\right)$ and $\tau_{2} \in C_{2}\left(C_{1}\right){ }^{31} C_{1}$ is the time-ordering contour from $-\infty$ to $+\infty$ and $C_{2}$ is the time-antiordering contour from $+\infty$ to $-\infty$. We find

$$
\begin{aligned}
\sum_{\text {int }}^{\lessgtr}\left(t_{1}, t_{2}\right)^{(2)}= & -2 J_{s d}^{2} \sum_{i, m, n}\left|\left\langle m\left|S^{i}\right| n\right\rangle\right|^{2} G_{0}^{\lessgtr}\left(t_{1}, t_{2}\right) \\
& \times\left[D_{0}^{\lessgtr}\left(t_{1}, t_{2}\right)\right]_{n, n}\left[D_{0}^{<}\left(t_{2}, t_{1}\right)\right]_{m, m} \\
= & -2 J_{s d}^{2} \sum_{i, m, n}\left|\left\langle m\left|S^{i}\right| n\right\rangle\right|^{2} G_{0}^{\lessgtr}\left(t_{1}, t_{2}\right) \\
& \times P_{n}\left(1-P_{m}\right) e^{ \pm i\left(\varepsilon_{m}-\varepsilon_{n}\right)\left(t_{1}-t_{2}\right)},
\end{aligned}
$$

where in the second step we have written explicitly $D_{0}^{\lessgtr}\left(t_{1}, t_{2}\right)$ in terms of the spin-level occupations $P_{n}=d_{n}^{\dagger} d_{n}$. The dependence of $\Sigma_{\text {int }}^{>}$over the energy $E$ can found by a simple Fourier transform

$$
\begin{aligned}
\Sigma_{\text {int }}^{\lessgtr}(E)^{(2)}= & -2 J_{s d}^{2} \sum_{i, m, n}\left|\left\langle m\left|S^{i}\right| n\right\rangle\right|^{2} P_{n}\left(1-P_{m}\right) \\
& \times G_{0}^{\lessgtr}\left(E \pm \Omega_{m n}\right),
\end{aligned}
$$

where $\Omega_{m n}=\varepsilon_{m}-\varepsilon_{n}$ and where the $+(-)$ symbol corresponds to $\Sigma^{<}\left(\Sigma^{>}\right)$.

Electron-spin scattering events are now fully described by $\left[\Sigma_{\text {int }}^{\lessgtr}(E)\right]$. In particular, Eq. (14) describes the process where an incoming electron with energy $E$ experiences a spin-flip process, which changes its energy by $\pm \Omega_{m n}$. This is the result of the electron-spin interaction with the local spins. Such a process is schematically represented in Fig. 2. Note that the probability for an excitation to occur is determined by the prefactors $\left.\left\langle m\left|S^{i}\right| n\right\rangle\right|^{2} P_{n}\left(1-P_{m}\right)$, i.e., by the state of the spin system.

We conclude this section by discussing the limits of validity of our perturbative expansion. On a first inspection of Eq. (14), one could conclude that the strength of the second-order interacting self-energy is solely dependent on $J_{s d}^{2}$. This will indeed result in a large perturbation parameter and thus in a possibly divergent perturbative expansion (see Table I for an estimate of the various parameters). However, both the lesser and greater Green's functions are proportional to the density of states of the scattering region $\rho$ [see Eq. (25)]. In the weak-coupling limit one has $\rho \sim \Gamma / \varepsilon_{0}^{2}$, where $\varepsilon_{0}$ is the on-site energy of the atom under consideration (the one under the tip) and $\Gamma$ is the total broadening due to the electrodes $\Gamma=\Gamma_{\text {tip-S }}+\Gamma_{\text {sub-s. }}$. As a consequence, we have that $\Sigma_{\text {int }}^{\lessgtr}(E)^{(2)} \sim \alpha J_{s d}$, where $\alpha=\rho J_{s d}$ is a dimensionless quantity. By continuing the expansion to the third order, one will find an additional contribution to the interacting self-energy proportional to $\alpha^{2} J_{s d}$, i.e., it will be discovered that the perturbation expansion parameter is the dimensionless quantity $\alpha$. The convergence of the perturbation series is then guaranteed by our weak-coupling condition, which essentially establishes that $\rho$ is small and then $\alpha \ll 1$.

\section{NONEQUILIBRIUM GREEN'S FUNCTION METHOD FOR ELECTRON TRANSPORT}

The transport properties of the device are described by using the NEGF method. This has been extensively described in the 
past. ${ }^{17-19}$ Here we only summarize the main concepts and we highlight the modifications associated to describing electronspin interaction. A two-probe device can be divided into three distinct regions, two semi-infinite leads which represent the STM tip and the underlying substrate and a central scattering region (see Fig. 1). The leads act as charge reservoirs, and they are characterized by their chemical potential, respectively,

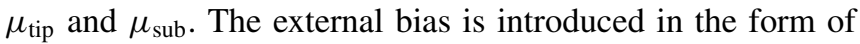
a relative shift (symmetric) of the two chemical potentials with respect to each other. The underluying assumption of the method is that under the external bias there is no rearrangement of the electronic structure of the leads, i.e., that electron screening in the leads is efficient. This simplifies the problem to that of calculating the retarded Green's function of the scattering region ${ }^{18,19,30}$ only:

$$
G(E)=\lim _{\eta \rightarrow 0}\left[(E-i \eta) I-H_{\mathrm{e}}-\Sigma(E)\right]^{-1} .
$$

Here $H_{\mathrm{e}}$ is the electronic part of the Hamiltonian [Eq. (1)] and $\Sigma(E)$ is the retarded self-energy, which incorporates the effects of the leads and of the inelastic interaction. In particular, $\Sigma(E)$ writes as

$$
\Sigma(E)=\Sigma_{\text {tip }}(E)+\Sigma_{\text {sub }}(E)+\Sigma_{\text {int }}(E),
$$

where $\Sigma_{\text {tip }}(E)$ and $\Sigma_{\text {sub }}(E)$ are, respectively, the STM tip and substrate self-energies, while $\Sigma_{\text {int }}$ is the scattering selfenergy describing the electron-spin interaction. The leads' self-energies can be written in terms of the surface Green's functions $\hat{g}_{\text {tip }}$ and $\hat{g}_{\text {sub }}$ and the coupling matrices between the leads and the scattering region $H_{\alpha-\mathrm{S}}(\alpha=$ tip, sub):

$$
\begin{gathered}
\Sigma_{\text {tip }}(E)=H_{\text {tip-S }}^{\dagger} \hat{g}_{\text {tip }}(E) H_{\text {tip-S }}, \\
\Sigma_{\text {sub }}(E)=H_{\text {sub-S }} \hat{g}_{\text {sub }}(E) H_{\text {sub-S }}^{\dagger} .
\end{gathered}
$$

The surface Green's functions can be found by first constructing the Green's function for an infinite system and then by applying the appropriate boundary conditions. ${ }^{32}$ For a single-site, nearest-neighbor, one-dimensional tight-binding chain, $\hat{g}_{\alpha}$ takes a simple close form

$$
\begin{gathered}
\hat{g}_{\text {tip }}(E)=\hat{g}_{\text {sub }}(E)=\frac{1}{\gamma_{0}} e^{i k(E),} \\
k(E)=\cos ^{-1}\left(\frac{E-\varepsilon_{0}}{2 \gamma_{0}}\right),
\end{gathered}
$$

where $k(E)$ and $\gamma_{0}$ are, respectively, the dispersion relation and the hopping parameter. We assume an equal hopping parameter in the leads and also the condition $\gamma_{0}>\varepsilon_{0}$. The interaction between the scattering region and the leads has the effect of broadening the discrete energy levels in the scattering region. The broadening functions write

$$
\Gamma_{\alpha}(E)=i\left[\Sigma_{\alpha}(E)-\Sigma_{\alpha}^{\dagger}(E)\right],
$$

where ( $\alpha=$ tip, sub). The resulting lesser and greater lead selfenergies are related to the broadening and to the population of the electrons and holes in the leads,

$$
\begin{gathered}
\Sigma_{\alpha}^{<}(E)=f_{\alpha}(E) \Gamma_{\alpha}(E), \\
\Sigma_{\alpha}^{>}(E)=\left[1-f_{\alpha}(E)\right] \Gamma_{\alpha}(E),
\end{gathered}
$$

where $f_{\alpha}(E)=1 /\left[1+\exp \left(\frac{E-\mu_{\alpha}}{k_{B} T}\right)\right]$ are the Fermi functions.
Finally, the retarded electron-spin scattering self-energy is found from the Hilbert transform ${ }^{30}$

$$
\begin{aligned}
\Sigma_{\text {int }}(E)= & \mathcal{P} \mathcal{V} \int_{-\infty}^{\infty} \frac{d E^{\prime}}{2 \pi} \frac{\Sigma_{\text {int }}^{>}\left(E^{\prime}\right)-\Sigma_{\text {int }}^{<}\left(E^{\prime}\right)}{E^{\prime}-E} \\
& -i \frac{\Sigma_{\text {int }}^{>}(E)-\Sigma_{\text {int }}^{<}(E)}{2},
\end{aligned}
$$

where $\mathcal{P} \mathcal{V}$ denotes the principal value and where the full expressions for $\Sigma_{\text {int }}^{>}(E)$ and $\Sigma_{\text {int }}^{<}(E)$ have been given in the previous section. Note that at variance with a similar expression for the self-energy describing electron-phonon scattering, in Eq. (24) there is no Hartree-like contribution. This usually describes polaronic effects and is known to cause the breakdown of the Born approximation for strong electron-phonon coupling. ${ }^{33}$

Additional quantities of interest which enter in the definition of the electron current, are the lesser and greater Green's functions $\left(G^{<}\right.$and $\left.G^{>}\right)$. Their diagonal elements respectively give the electron and hole density in the scattering region. These are defined by

$$
G^{\lessgtr}(E)=G(E)\left[\Sigma^{\succ}(E)\right] G^{\dagger}(E),
$$

where

$$
\Sigma>(E)=\Sigma_{\text {tip }}^{\lessgtr}(E)+\Sigma_{\text {sub }}^{\lessgtr}(E)+\Sigma_{\text {int }}^{\lessgtr}(E) .
$$

As already mentioned, the application of a potential difference $V$ across the device produces a shift in the tip and substrate chemical potentials. By assuming, as done here, that the tip and the substrate share the same Fermi level $E_{\mathrm{F}}$, then we have $\mu_{\text {tip }}=E_{\mathrm{F}}+e V / 2$ and $\mu_{\text {sub }}=E_{\mathrm{F}}+e V / 2$. This is equivalent to replacing the energy in the leads with $E \rightarrow E \pm e V / 2(e$ is the electron charge). Finally the current can then be calculated for a range of values of $V$ at any of the leads $\alpha$ as

$$
I_{\alpha}=\int_{-\infty}^{+\infty} \bar{I}_{\alpha}(E) d E,
$$

$$
\bar{I}_{\alpha}(E)=\frac{e}{h} \operatorname{Tr}\left\{\left[\Sigma_{\alpha}^{>}(E) G^{<}(E)\right]-\left[\Sigma_{\alpha}^{<}(E) G^{>}(E)\right]\right\} .
$$

The conductance is found by differentiating the above expression with respect to the bias.

In concluding this section we would like to discuss the expected magnitude of the inelastic contribution to the conductance with respect to the elastic one. The ratio between two such contributions essentially corresponds to the ratio between the interacting and the noninteracting $\left(J_{s d}=0\right)$ Green's functions. A simple calculation shows that the unperturbed Green's function differs by a factor of $\Sigma_{\text {int }} / \Gamma$ from the fully interacting one. In the previous section we showed that to the second order $\Sigma_{\text {int }}^{(2)} \sim \alpha J_{s d}$. Therefore, to the second order, the ratio between the elastic and inelastic contributions to the conductance turns out to be proportional to the dimensionless factor $\alpha\left(J_{s d} / \Gamma\right) \sim\left(J_{s d} / \varepsilon_{0}\right)^{2}$. Analogously, the third-order contribution will account for a factor $\alpha^{2}\left(J_{s d} / \Gamma\right)$. With this in hand and by looking at the parameters in Table I, we can conclude that the contribution originating from the secondorder expansion will be significant, while that from the third 
TABLE I. Empirical parameters used in the numerical simulations presented in this work and their assigned numerical values. In the rightmost column we report the source of a particular estimate. "Channel" here means the scattering region.

\begin{tabular}{lccc}
\hline \hline Quantity & Symbol & Value & Origin \\
\hline Atomic spin & $S$ & $5 / 2$ & Exp. (Ref. 7) \\
Temperature & $T$ & $0.6 \mathrm{~K}$ & Exp. (Ref. 7) \\
$d-d$ exchange & $J_{d d}$ & $+6.2 \mathrm{meV}$ & Exp. (Ref. 7) \\
$s-d$ exchange & $J_{s d}$ & $+500 \mathrm{meV}$ & DFT (Refs. 34 and 35) \\
Fermi energy & $E_{\mathrm{F}}$ & $0 \mathrm{meV}$ & DFT (Ref. 34) \\
Lead hopping integral & $\gamma_{0}$ & $10000 \mathrm{meV}$ & DFT (Ref. 34) \\
Channel on-site energy & $\varepsilon_{0}$ & $1000 \mathrm{meV}$ & DFT (Ref. 34) \\
Tip-channel hopping & $\gamma_{\text {tip-S }}$ & $50 \mathrm{meV}$ & DFT (Ref. 34) \\
Substrate-channel hopping & $\gamma_{\text {sub-s }}$ & $500 \mathrm{meV}$ & DFT (Ref. 34) \\
Axial anisotropy & $D$ & $-0.037 \mathrm{meV}$ & Exp. (Ref. 5) \\
Transverse anisotropy & $E$ & $0.007 \mathrm{meV}$ & Exp. (Ref. 5) \\
\hline \hline
\end{tabular}

order will be minute. As a consequence, our strategy to stop the expansion to the second order appears well justified.

\section{RESULTS}

The entire procedure outlined in the previous two sections is now implemented numerically in order to calculate the $I-V$ characteristics and the conductance spectra $(d I / d V-V)$ of some selected magnetic nanostructures. In particular, we aim at reproducing the $d I / d V-V$ curve observed through Mn monoatomic chains deposited on thin $\mathrm{CuN}$ insulating atomic layers, as described in Ref. 7. Clearly our one-dimensional (1D) model does not include all the details involved in the actual SF-IETS STM experiments, but we argue here that it contains already all the ingredients to reproduce the main experimental features.

In Table I we list all the parameters used in our simulations and their assigned values. These have been either inferred from the experiments (Exp) (Ref. 7) or have been estimated from density functional theory (DFT) calculations. ${ }^{34}$ The local Mn spin is set to be $5 / 2$, as proposed in the original experimental works, ${ }^{5,7}$ confirmed by DFT calculations, ${ }^{34}$ and expected from the nominal $\mathrm{Mn}$ valence. The spin-spin coupling parameter $J_{d d}$ corresponds to an antiferromagnetic order between the neighboring Mn spins, a feature verified in the experimental conductance spectra. The lead on-site energy is suitably set to zero and simply defines the reference potential. We also note that the value for $J_{s d}$ is determined from theory to be of the order of $500 \mathrm{meV}{ }^{34,35}$ Although we employ a zero-temperature formalism, we nevertheless evaluate the Fermi functions of the leads at the small temperature of $0.6 \mathrm{~K}$. This allows us to include minor thermal smearing of the electron gas in the leads and consequently of the conductance profile. Finally we notice that the scattering region is expected to be significantly more strongly coupled to the substrate than to the tip. This essentially means that the spin population in the scattering region remains effectively in equilibrium ${ }^{15}$ with the substrate, i.e., there is no accumulation of spin excitations between two inelastic tunneling events.

Figure 3 shows the calculated conductance spectra normalized against the elastic contribution $G_{\mathrm{el}}$ [this is the conductance calculated for $\Sigma_{\mathrm{S}}(E)=0$ ] for $N$-atom-long $\mathrm{Mn}$ chains $(N \leqslant 4)$ in no external magnetic field. From the figure we observe that the conductance increase due to the inelastic contribution is of the order of $1 / 4$ (for $N=2$, where the amplitudes of the spin matrix elements of the self-energy are approximately unity). For our choice of parameters the scaling factor is $\left(J_{s d} / \varepsilon_{0}\right)^{2}=1 / 4$. Such a good agreement validates our analysis on the limits of the perturbation expansion. We can then predict that the corresponding conductance increase that might originate from third-order contributions [these scale as $\left.\alpha^{2}\left(J_{s d} / \Gamma\right)\right]$ is significantly smaller. This estimate justifies our original choice of stopping the perturbation expansion to the second order.

In the case of $N=3$ and $N=4$ the spectrum is calculated for the STM tip placed above the second atom in the chain. In Fig. 3 we also show that by placing the tip above the first atom in the chain for the $N=3$ case we drastically decrease the conductance step at $\sim 17 \mathrm{meV}$. A similar occurrence is seen in the conductance step for $N=4$. In general, we chose to position the tip over the second atom in order to reproduce the experimental spectra more accurately. From the figure it is clear that all the qualitative experimental findings

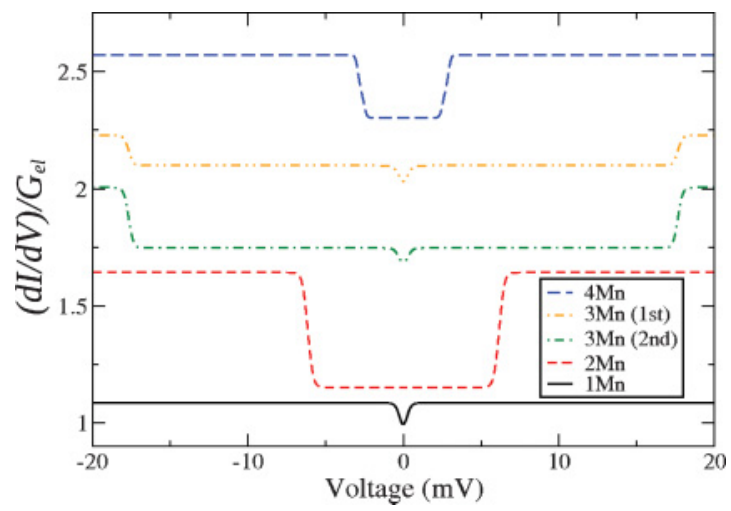

FIG. 3. (Color online) Normalized conductance spectra for Mn chains of different lengths $N$. The various spectra, except for $N=1$, are offset for clarity. The tip is placed above the second atom of the chain for chains with $N>2$ and also over the first atom in the case of $N=3$. We notice the strong dependence of the spectra on the chain parity. 
are correctly reproduced. In particular, we notice the strong dependence of the conductance profile over the parity of the chains, with chains comprising an odd number of atoms (odd chains) exhibiting a conductance dip at approximately $V=0$, which is absent for even chains. It is worth noting, however, that the spectra for $N=3$ and $N=4$ contrast slightly with the experimental spectra, which are asymmetric with respect to the bias and also exhibit some slope at the conductance steps. We believe that these features are a manifestation of nonequilibrium effects ${ }^{15}$ in the spin system, i.e., to the fact that the local spins do not relax instantaneously to their ground state. These effects are not included at the present level of the theory.

The ground state of each odd chain has a net total spin $S_{\text {tot }}=$ $5 / 2$. This is affected by the transverse and axial anisotropy, which lift the ground-state degeneracy and allow a transition between the ground state and the first spin-flip excited state to occur. The excitation of such a transition results in a conductance step at a voltage corresponding to the transition energy. Since the anisotropy energies are small (see Table I), the excitation energy is small as well, and the feature in the conductance profile appears near $V=0$. In contrast, the even chains do not show any zero-bias excitation, since the ground state is a singlet and does not carry a magnetic moment. This is direct proof that the magnetic interaction between $\mathrm{Mn}$ ions is antiferromagnetic. Should this have been ferromagnetic, even chains would also have shown zero-bias anomalies.

As mentioned above, the conductance steps encountered at approximately $V=0$ correspond to spin-flip events, i.e., to electron scattering processes that produce the transition $|m=5 / 2\rangle \rightarrow|m=3 / 2\rangle$ ( $m$ is the magnetic quantum number) but that also preserve the total spin $S_{\text {tot }}=5 / 2$. The first net spin changing transition occurs for $N=2$ and corresponds to the large conductance step found at $V=J_{d d} / e=6.2 \mathrm{mV}$. This is investigated in more detail in Fig. 4, where we also include the dimer's spectrum calculated when a magnetic field is applied along the $z$ direction. The effect of the magnetic field is that of splitting the single excitation line into three distinct conductance steps. In this case, in fact, the antiferromagnetic dimer has a singlet $\left(S_{\mathrm{tot}}=0\right)$ ground state and a triplet $\left(S_{\text {tot }}=1\right)$ first excited state. Therefore, an excitation from the ground state to the first excited state changes the net spin of the dimer. When a magnetic field is applied the degeneracy of the triplet excited state is lifted and excitations having the three Zeeman-split levels as final states are possible. This produces the threefold splitting of the conductance steps, as seen to agree well with experiment in Fig. 4.

Figure 5 shows the conductance spectrum for the trimer $(N=3)$. This chain exhibits similar trends to those found for the dimer as a magnetic field is applied, i.e., there is a Zeeman split of the zero-field conductance steps. From the figure one may notice that for both the theoretical (A) and experimental (B) data there is a shift of the principle step to lower voltages as the magnetic field is increased. We remark here that in order to recreate the conductance profile of the trimer, additional ferromagnetic second-nearest-neighbor interaction between the local spins at the edge of the chain must be included in the model. ${ }^{11}$ The magnitude of this additional exchange parameter is approximately half of that of $J_{d d}$. The inclusion of such a second-nearest-neighbor coupling constant
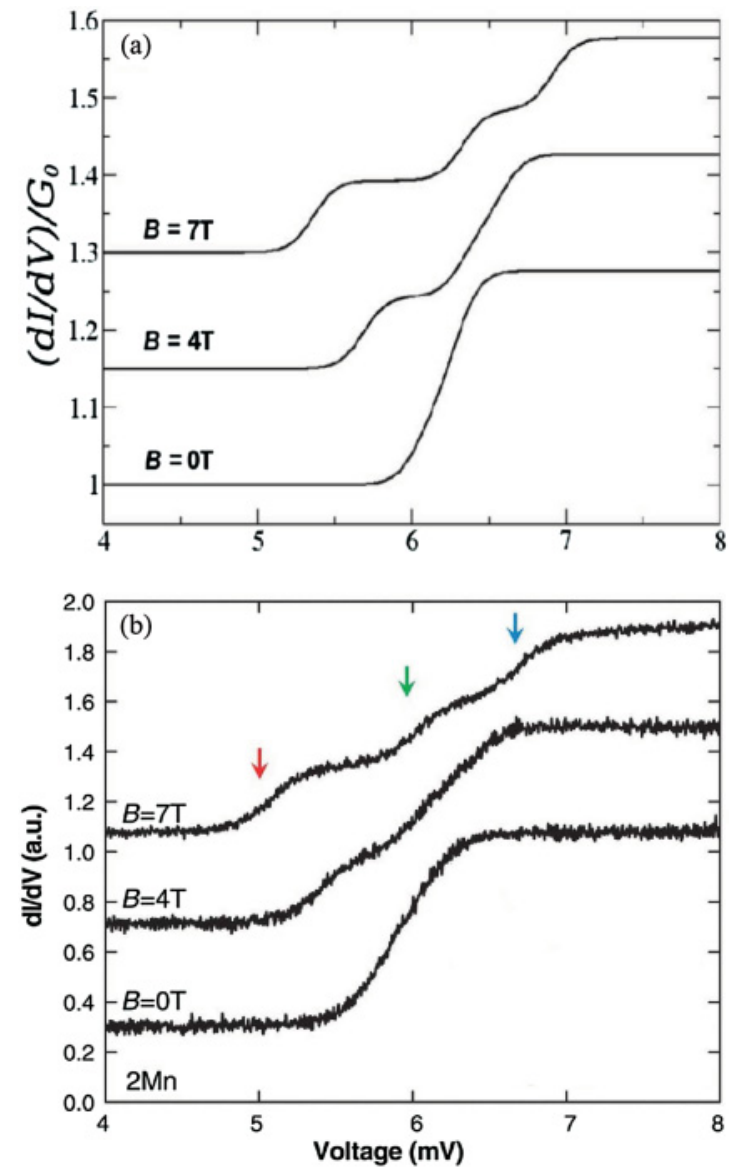

FIG. 4. (Color online) Theoretical (A) and experimental (B) conductance spectra for the $N=2$ chains in a finite external magnetic field. For $\mathbf{B} \neq 0$ we note a splitting of the conductance steps, corresponding to the Zeeman split of the final triplet excited state. This is observed in the experiments of Ref. 7, which are also reported in (B).

changes the position of the conductance step from a second excited state at $27 \mathrm{mV}$ to a first excited state at $16 \mathrm{mV}$. This correction is also included in the calculations for $N=4$ (see Fig. 3), again giving good agreement with experiments.

In all of the spectra investigated, the most striking agreement with the experiments concerns the correct prediction of the SF-IETS excitation voltages. In particular, not all the possible spin excitations feature in the $d I / d V-V$ curve as a result of spin-selection rules. A careful analysis of the inelastic process, as outlined in the first section, reveals why some excitations occur and why some other do not. In particular, we note that the proper selection rules for transitions are governed by the prefactor in the self-energy $\left|\left\langle m\left|S^{i}\right| n\right\rangle\right|^{2}$ [Eq. (14)]. This effectively selects which excitations are more probable to occur. For example, the full energy spectrum of the trimer has $6^{3}=216$ eigenvalues, but only a small portion of these eigenvalues will contribute significantly to the scattering self-energy. Consequently, only a few transitions will have influence on the conductance spectrum. This, for instance, explains why the first conductance step of the dimer is considerably larger than that of other chains of different lengths. In fact, our calculations show that a transition from the singlet ground state $(S=0)$ to any of the triplet excited states 

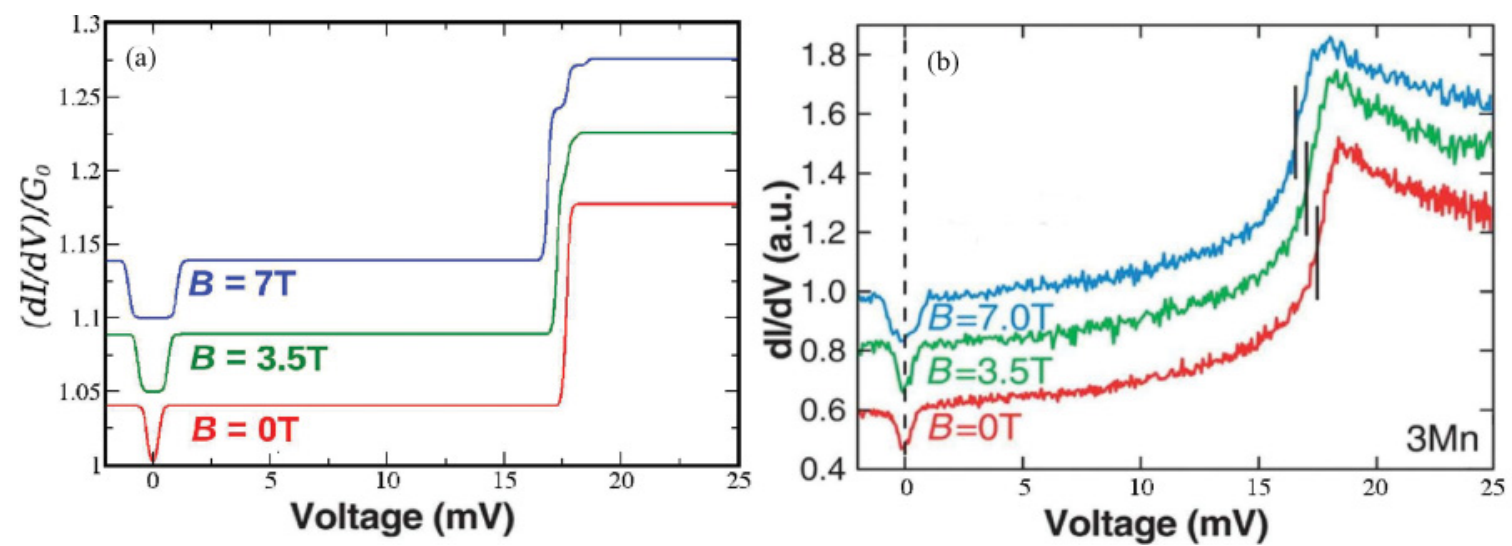

FIG. 5. (Color online) Theoretical (A) and experimental (B) conductance spectra for the $N=3$ trimer at different magnetic fields. We notice the shift of the first conductance step transition to lower voltages as the magnetic field increases. This is in good agreement with STM experiments of Ref. 7, which are reproduced here in (B).

( $S=1 ; m=-1,0,+1$ ) has equal probability. This results in a conductance step which is approximately three times larger than any single spin-flip event that occurs in an odd chain.

\section{CONCLUSIONS}

In conclusion, we have formulated a theory of electron transport through magnetic nanostructures based on the NEGF formalism and including inelastic spin scattering. We have first explicitly derived an expression for the interacting selfenergy at the level of the first Born approximation in the electron-spin interaction. This has been used to calculate the current-voltage and the conductance-voltage curves for a 1D system of magnetically coupled spins at finite bias and in a magnetic field. Our results reproduce well the features of recent SF-IETS experiments for 1D Mn chains. Most notably, the severe dependence of the conductance spectra on the chain parity and the selection-rule suppression of certain transitions is a direct outcome of the theory. Our NEGF approach is therefore a valid alternative to master-equation-based schemes, with the advantage that it can be scaled up to larger systems and combined with more sophisticated electronic structure theories.

\section{ACKNOWLEDGMENTS}

This work is sponsored by the Irish Research Council for Science, Engineering \& Technology (IRCSET). N.B. and S.S. thank the Science Foundation of Ireland (Grant No. 08/ERA/I1759) and CRANN for financial support. Computational resources have been provided by the Trinity Centre for High Performance Computing (TCHPC). We wish to thank Dr. Cyrus Hirjibehedin for the experimental data shown in Figs. 4 and 5.
${ }^{1}$ R. C. Jaklevic and J. Lambe, Phys. Rev. Lett. 17, 1139 (1966).

${ }^{2}$ B. C. Stipe, M. A. Rezaei, and W. Ho, Science 280, 1732 (1998).

${ }^{3}$ A. J. Heinrich, J. A. Gupta, C. P. Lutz, and D. M. Eigler, Science 306, 466 (2004).

${ }^{4}$ D. M. Eigler and E. K. Schweizer, Nature (London) 344, 524 (1990).

${ }^{5}$ C. F. Hirjibehedin, C. Lin, A. F. Otte, M. Ternes, C. P. Lutz, B. A. Jones, and A. J. Heinrich, Science 317, 1199 (2007).

${ }^{6}$ A. F. Otte, M. Ternes, S. Loth, C. P. Lutz, C. F. Hirjibehedin, and A. J. Heinrich, Phys. Rev. Lett. 103, 107203 (2009).

${ }^{7}$ C. F. Hirjibehedin, C P. Lutz, and A. J. Heinrich, Science 312, 1021 (2006).

${ }^{8}$ X. Chen, Y.-S. Fu, S.-H. Ji, T. Zhang, P. Cheng, X.-C. Ma, X.-L. Zou, W.-H. Duan, J.-F. Jia, and Q.-K. Xue, Phys. Rev. Lett. 101, 197208 (2008).

${ }^{9}$ C. Romeike, M. R. Wegewijs, and H. Schoeller, Phys. Rev. Lett. 96, 196805 (2006).

${ }^{10}$ J. Tersoff and D. R. Hamann, Phys. Rev. Lett. 50, 1998 (1983).

${ }^{11}$ J. Fernandez-Rossier, Phys. Rev. Lett. 102, 256802 (2009).
${ }^{12}$ J. Fransson, O. Eriksson, and A. V. Balatsky, Phys. Rev. B 81, 115454 (2010).

${ }^{13}$ N. Lorente and J. P. Gauyacq, Phys. Rev. Lett. 103, 176601 (2009).

${ }^{14}$ M. Persson, Phys. Rev. Lett. 103, 050801 (2009).

${ }^{15}$ B. Sothmann and J. König, New J. Phys. 12, 083028 (2010).

${ }^{16}$ F. Delgado, J. J. Palacios, and J. Fernandez-Rossier, Phys. Rev. Lett. 104, 026601 (2010).

${ }^{17}$ L. V. Keldysh, Sov. Phys. JETP 20, 1018 (1965).

${ }^{18}$ S. Datta, Electronic Transport in Mesoscopic Systems (Cambridge University Press, Cambridge, UK, 1995).

${ }^{19}$ A. R. Rocha, V. M. Garcia-Suarez, S. Bailey, C. Lambert, J. Ferrer, and S. Sanvito, Phys. Rev. B 73, 085414 (2006).

${ }^{20}$ I. Rungger and S. Sanvito, Phys. Rev. B 78, 035407 (2008).

${ }^{21}$ C. D. Pemmaraju, I. Rungger, X. Chen, A. R. Rocha, and S. Sanvito, Phys. Rev. B 82, 125426 (2010).

${ }^{22}$ M. Galperin, M. A. Ratner, and A. Nitzan, J. Chem. Phys. 121, 11965 (2004).

${ }^{23}$ V. P. Antropov, M. I. Katsnelson, M. van Schilfgaarde, and B. N. Harmon, Phys. Rev. Lett. 79, 729 (1997). 
${ }^{24}$ M. Stamenova, T. N. Todorov, and S. Sanvito, Phys. Rev. B 77, 054439 (2008).

${ }^{25}$ K. Yosida, Theory of Magnetism (Springer, Berlin, 1996).

${ }^{26}$ X. Wang, C. D. Spataru, M. S. Hybertsen, and A. J. Millis, Phys. Rev. B 77, 045119 (2008).

${ }^{27}$ G. D. Mahan, Many-Particle Physics, 2nd ed. (Plenum, New York, 1990).

${ }^{28}$ T. Frederiksen, M. Brandbyge, N. Lorente, and A. P. Jauho, J. Comput. Electron. 3, 423 (2004).

${ }^{29}$ P. Hyldgaard, S. Hershfield, J. H. Davies, and J. W. Wilkins, Ann. Phys. (NY) 236, 1 (1994).
${ }^{30}$ A. A. Yanik, G. Klimeck, and S. Datta, Phys. Rev. B 76, 045213 (2007).

${ }^{31}$ H. Haug and A. P. Jauho, Quantum Kinetics in Transport and Optics of Semiconductors (Springer, Berlin, 1996).

${ }^{32}$ S. Sanvito, C. J. Lambert, J. H. Jefferson, and A. M. Bratkovsky, Phys. Rev. B 59, 11936 (1999).

${ }^{33}$ W. Lee, N. Jean, and S. Sanvito, Phys. Rev. B 79, 085120 (2009).

${ }^{34}$ R. Zitko and T. Pruschke, New J. Phys. 12, 063040 (2010).

${ }^{35}$ P. Lucignano, R. Mazzarello, A. Smogunov, M. Fabrizio, and E. Tosatti, Nat. Mater. 8, 563 (2009). 